\title{
Priors in a Bayesian Audit: How Integration of Existing Information into the Prior Distribution Can Improve Audit Transparency and Efficiency
}

\begin{abstract}
Auditors often have prior information about the auditee before starting the substantive testing phase. We show that applying Bayesian statistics in substantive testing allows for integration of this information into the statistical analysis through the prior distribution. For example, an auditor might have performed an audit last year, they might have information on certain controls in place, or they might have performed analytical procedures in an earlier stage of the audit. Incorporating this information directly in the statistical procedure enables auditors to tailor their sampling plan to the auditee, thereby increasing audit transparency and efficiency. However, defining a suitable prior distribution can be difficult because what constitutes a suitable prior depends on the specifics of the audit and the auditee. To help the auditor in constructing a prior distribution we introduce five methodologies, consider their pros and cons, and give examples of how to apply them in practice.
\end{abstract}

Keywords: audit, Bayesian statistics, financial statements, prior distribution.

\section{INTRODUCTION}

A financial audit is an inspection of an organization's financial statements before they are released to the public. In the audit report, the auditor presents their opinion on the fairness of these statements to inform stakeholders of the organization about its current financial situation. An organization's financial statements are derived from several large populations of transactions (Neter and James 1975; Neter and Loebbecke 1977; Ramage et al. 1979; Titera 2013) that, until a few decades ago, were all assessed in detail (Power 1992). Nowadays, technological advancements (e.g., Big Data, Artificial Intelligence) theoretically allow auditors to inspect these large (digital) populations completely. However, in most businesses, the data- and business process quality would make a complete inspection result in a high number of seemingly irrelevant audit findings (Brown-Liburd et al. 2015). Moreover, Early (2015) and Gepp et al. (2018) argue that these techniques are only slowly progressing in the 
audit practice. Recently, Yoon and Pierce (2020) argue that such analytical procedures and statistical sampling both have unique benefits, and that they are best used to complement each other. Statistical sampling therefore remains a much-used technique due to its efficiency in larger populations (Christensen, Elder, and Glover 2015; Hitzig 1995; IAASB 2018, Maingot and Quon 2009; Srivenkataramana 2018; van der Nest et al. 2015). After all, statistical sampling enables the auditor to infer a conclusion about a certain characteristic of the population based on only a small subset of this population.

The challenge that auditors routinely face is to tailor their statistical procedures to the specific situation of the audit and the auditee (Brivot et al. 2018; Coram et al. 2003; Lombardi et al. 2014). Due to variability in organizations' administrative systems, controls, or possible malicious intent, no two organizations share the same quality of financial reporting and, thus, can be audited in the exact same manner (Beaulieu 2001; Kachelmeier et al. 2014). In this article, we show that Bayesian statistics allows for this tailoring, arguably resulting in a more transparent and more efficient audit.

The International Standards on Auditing (ISA; IAASB 2018) prescribe that the auditor can use statistical sampling to quantify the risk of an incorrect judgment resulting from their substantive testing procedures ${ }^{1}$. To conduct statistical sampling, ISA 530 mandates "the use of probability theory to evaluate sample results" (IAASB 2018). While in probability theory there are two main schools of thought, Bayesianism and frequentism (Dienes 2011; Wagenmakers, Lee, Lodewyckx, and Iverson 2008), auditing firms have mostly relied on frequentist methods to design their statistical sampling procedures and substantiate their conclusions (Christensen et al. 2015). In addition, frequentist estimation and testing have a crucial role in the accounting curriculum of most universities (see for example Abeysekera

\footnotetext{
${ }^{1}$ Note that the audit standards facilitate but do not mandate the use of statistical sampling.
} 
2015, and Lee et al. 2018). Although the standards' requirements for statistical sampling cannot be found exclusively in frequentist methods, it is clear that these methods currently dominate audit theory and practice. However, Bayesian methods that use probability theory to evaluate sample results may legitimately be used to make an inference in substantive testing.

The auditing standards prescribe that auditors are allowed to reduce the quantity of evidence that is required from substantive testing if they have existing information on an auditee that indicates a low risk profile (ISA 530, IAASB 2018). For the statistical analysis, this usually translates into a reduced sample size for auditees with a lower risk profile. However, within the frequentist framework, it is not possible to incorporate this existing information into the statistical methodology, except on an ad-hoc basis (Wagenmakers, Lee, Lodewyckx, and Iverson 2008). Frequentist methods allow the auditor to modify their audit procedures on the basis of existing information, but they do not allow the auditor to coherently incorporate this information into the analysis. Because it is unclear in a frequentist analysis what the mathematical relationship is between the existing information and the sample information it is said to be incoherent (Lindley 2004). As a result of this incoherency, frequentist substantive testing procedures often expose the auditor to an audit risk that is greater than is desired (Jiambalvo and Waller 1984; Kinney 1983; Stewart 2013, pp. 58).

In contrast to frequentist statistics, Bayesian statistics has been continuously advocated as a means to integrate existing information into substantive testing in a coherent manner (Beck et al. 1985; Corless 1972; Crosby 1980; Felix 1976; Godfrey and Neter 1984; Laws and O’Hagan 2002; Meeden 2003; Sahu and Smith 2006; Tsui et al. 1985). More concretely, in the Bayesian approach, the auditor quantifies their existing information in a prior probability distribution such that it captures the information available to them. Since the prior distribution contains information that is specific to the auditee and the population, it allows for an informed and tailor-made point of departure for substantive testing. Bayesian statistics uses 
the rules of probability theory to revise the information in the prior distribution in light of the observed sample. This approach to revising information highlights the cumulative character of a Bayesian analysis, one on which many scholars and practitioners have agreed is appropriate in an audit context (Kinney 1975; Stewart 2013) because an audit itself is a continuous process (Leslie 1984). Another argument why Bayesian methods are appropriate for auditors is that the audit standards describe an audit along the lines of that same philosophy. For example, ISA 330 (paragraph A60) states that "an audit of financial statements is a cumulative and iterative process" (IAASB 2018). Thus, one could argue that the Bayesian approach fits well with the audit standards and the goal of the auditor.

Despite these advantages, the use of Bayesian methods in the audit is scarce. A potential reason for this scarcity is that auditors need to translate existing audit information into a prior probability distribution, which is not necessarily part of the expertise of an auditor (Corless 1972; Felix 1976; Martel-Escobar et al. 2018; Stewart 2013). First, determining the type of audit information to incorporate into the prior distribution can be difficult because what information might be incorporated depends on the situation at hand. Second, the translation from relevant audit information into a prior probability distribution is perceived as difficult (Abdolmohammadi 1985; Abdolmohammadi 1987; Corless 1972). Nevertheless, by overcoming these hurdles the auditor can build upon existing information in a coherent manner, resulting in concrete advantages — such as a more accurate estimation of the population misstatement (Knoblett 1970) and audit risk (Stewart 2013), a potential reduction in sample size, and formalized predictions - that can increase efficiency and transparency in audit sampling. Furthermore, it has been shown in earlier studies based on the well-known audit populations from Neter and Loebbecke (1975) that Bayesian methods result in upper bounds that achieve nominal coverage (Chan and Smieliauskas 1990; Swinamer, Lesperance, and Will 2007). To increase the feasibility of Bayesian methods in the audit practice and to 
make these tangible advantages of Bayesian statistics more easily available for auditors, we will introduce five methods for incorporating existing audit information into a prior probability distribution. Please note that we do not see these Bayesian methods as a replacement for frequentist methods, but rather as an efficient addition to the auditor's statistical toolbox if needed.

The structure of this article is as follows. In section two the three building blocks of Bayesian inference are introduced: the prior distribution, the likelihood, and the posterior distribution. Next, section three discusses the pros and cons of using a prior distribution in an audit context. In section four, five methods of constructing a prior distribution are discussed. The last section presents our concluding comments.

\section{THE BAYESIAN APPROACH TO AUDIT SAMPLING}

In audit sampling, the goal of the auditor is to make a statement about a certain characteristic, $\theta$, of the population. Generally, the auditor does not inspect the entire population but only a sample, $y$, from this population. As a consequence, the information about $\theta$ from the sample is extrapolated to the population, introducing uncertainty and a probabilistic statement about $\theta$.

The Bayesian way of making a probability statement about the characteristic $\theta$, given the sample $y$, is through the posterior density $p(\theta \mid y)$. The posterior density is defined through Bayes' theorem as the product of two densities, the prior density $p(\theta)$ and the likelihood function $l(y \mid \theta)$, conditioned on the value of the sample $y$. Because the marginal probability of the data $p(y)$ is not dependent on $\theta$ and with a fixed sample it is a constant, the computation of the posterior density is often shown as: 


$$
\underbrace{p(\theta \mid y)}_{\text {Posterior }} \propto \underbrace{l(y \mid \theta)}_{\text {Likelihood }} \times \underbrace{p(\theta)}_{\text {Prior }}
$$

As Equation 1 illustrates, Bayes' theorem implies that the information in the prior distribution is combined with the information in the sample to form the posterior distribution. In the following subsections, we will further elaborate on the prior distribution, the likelihood, and the posterior distribution.

\section{The Prior Distribution}

The prior distribution $p(\theta)$ reflects the auditor's existing information about $\theta$ before seeing any information from a sample. An adequate prior distribution assigns a relative plausibility of occurrence to every possible value of $\theta$ such that the probability across all possible values of $\theta$ is equal to one. Which values of $\theta$ are possible depends on the audit question at hand, and the auditor must specify the family of the prior distribution accordingly.

For example, in monetary unit sampling (MUS) the goal is to estimate the amount of misstatement in the population, and monetary units (e.g., individual dollars) are selected and evaluated as individual units. The possible values of the total misstatement amount $\theta$ for a population of $M$ monetary units therefore lie in the interval $[0 ; M]$ and the data are generally assumed to be generated by a Poisson distribution. A gamma prior distribution is a common choice for MUS (Stewart 2013; Stewart, Strijbosch, Moors, and van Batenburg 2007) since it remains a gamma distribution when updated by the information in the data. However, Broeze (2006) shows that using a beta distribution as a prior distribution for MUS is also appropriate when using the proportional errors (i.e., taints) of the transactions when evaluating the sample. In the remainder of this article, we will demonstrate the five methods for constructing a prior distribution using a beta distribution because it is easy to explain. However, because in some cases proportional errors are unlikely to occur, we included a discussion of the gamma distribution in the appendix to this article. 
Because the total probability that a prior distribution assigns to all possible values of $\theta$ is equal to one the prior distribution is a probability distribution, and the auditor can summarize their prior knowledge about $\theta$ by calculating location measures such as the mean, median, and mode of the distribution. For example, the prior mode is the most likely value of $\theta$ before the sample is analyzed, and the prior mean is the expected value of $\theta$ before the sample is analyzed. Similarly, the auditor can summarize the spread of the prior distribution with an $x$-percent interval that ranges from the $(100-x) / 2^{\text {th }}$ percentile of the prior distribution to the $(100+x) / 2^{\text {th }}$ percentile. For example, the 90 percent interval $\left[\theta_{.05} ; \theta_{.95}\right]$ implies that, with a probability of 90 percent, the population characteristic $\theta$ lies between the values $\theta_{.05}$ and $\theta_{.95}$. Similarly, the prior distribution can also be interpreted with respect to the value $\theta_{.95}$ as stating that with 95 percent probability, the value of $\theta$ is lower than $\theta_{.95}$. Therefore the value $\theta_{.95}$ can be interpreted as a 95 percent upper bound since 95 percent of the probability mass lies below this value.

In the case of a beta prior distribution, a useful interpretation is that the auditor can interpret the information in the prior distribution as equivalent in information to that of an earlier sample of $n$ transactions containing $k$ errors (Crosby 1981, Steele 1992). Since the auditor usually assumes these earlier samples to be correct, they may be deducted from the current intended sample size, increasing audit efficiency (Stewart 2013; Touw and Hoogduin 2012).

To put the beta prior distribution in a more concrete context, consider an example from a standard financial audit in which the characteristic of interest $\theta$ is generally the misstatement in the population. Suppose an auditor specifies a $\operatorname{Beta}(1,1)$ prior distribution on $\theta$ (Figure 1), implying that every value of the (proportion of) misstatement is equally likely to occur a priori. Because this prior distribution is flat, the interpretation of this prior in 
terms of a prior sample is that the information contained in this prior is the information from a sample of size zero.

\section{The Likelihood}

The likelihood function $l(y \mid \theta)$ reflects the information that the observed data contain about the population characteristic $\theta$. It quantifies the likelihood of the sample outcomes occurring under specific values of $\theta$ (Etz 2018).

Returning to the previous example with the $\operatorname{Beta}(1,1)$ prior distribution, the likelihood function of observing $k$ misstatements in a sample of size $n$, with an underlying error rate parameter $\theta \in[0,1]$ is $\operatorname{Binomial}(k \mid n, \theta)$. Suppose that the auditor analyzes a sample of $n=58$ observations, in which they find $k=0$ misstatements. This implies that the likelihood function of this sample is $\operatorname{Binomial}(k=0 \mid n=58, \theta)$.

\section{The Posterior Distribution}

The posterior distribution $p(\theta \mid y)$ reflects the auditor's updated knowledge about the characteristic $\theta$ after having inspected a sample $y$ from the population. The posterior distribution follows from the fact that each candidate value of $\theta$ from the prior distribution induces a prediction about the observed data. Comparing the quality of these predictions from the prior distribution with the actual observed data induces a prediction error. Given the inspected sample $y$, some values of $\theta$ have a lower prediction error than other values. It is this prediction error that holds information about the extent to which the data ought to adjust the plausibility of different values for $\theta$, since the prediction error measures the discrepancy between the auditor's prior information and the information from the sample. Bayes' rule stipulates that values of $\theta$ that predict the sample relatively well receive a boost in plausibility, whereas values of $\theta$ that predict the sample relatively poorly suffer a decline. By assessing predictive accuracy for the candidate values of $\theta$ the prior distribution is updated to 
the posterior distribution, which consequently contains all information about $\theta$ that is available to the auditor.

Because the posterior distribution is a probability distribution the updated knowledge about $\theta$ can be assessed by location measures such as the mean, median, and mode of the posterior distribution. For example, the auditor can make a statement about the most likely error in the population by looking at the mode of the posterior distribution. Similarly, percentiles of the posterior distribution can be interpreted in terms of probability (Hoekstra, Morey, Rouder, and Wagenmakers 2014; Kruschke and Liddell 2017). For instance, a Bayesian auditor will typically say that, with 95 percent certainty, the maximum misstatement in the population is lower than the 95 th percentile of the posterior distribution, $\theta_{.95}$, since 95 percent of the probability mass lies below this value of $\theta$.

In Figure 1 the mode of the posterior distribution is $\frac{0}{58}=0$, implying that the most likely misstatement in the population is zero percent. The posterior mean is 0.017 , implying that the expected misstatement in the population is 1.7 percent. The 95 th percentile of the posterior distribution lies at 0.049 meaning that, with 95 percent probability, the maximum misstatement in the population is lower than 4.9 percent.

\section{PROS AND CONS OF THE PRIOR DISTRIBUTION}

Using the prior distribution as a point of departure for substantive testing increases transparency and efficiency for both the auditee and the auditor. However, this comes at the cost of justifying the prior distribution.

\section{Pro 1: The Prior Distribution Increases Transparency for the Auditor}


Currently, when the auditor wants to incorporate existing information into their frequentist analysis, they must do so in a manner that is not fully transparent. For instance, the auditor can "use the Audit Risk Model (ARM) to subjectively multiply the risk of material misstatement by the risk of incorrect acceptance of an hypothesis to arrive at an incoherent hybrid overall audit risk" (Stewart 2013, pp. 24).

Bayes' theorem, which explains how existing information is revised by sample evidence, can coherently incorporate this existing information in the prior distribution and update it using the observed sample outcomes. The use of Bayes' rule to intuitively show how the information in the prior distribution is updated by the sample to the information in the posterior distribution has the potential to improve transparency towards auditors.

\section{Pro 2: The Prior Distribution Increases Transparency Towards Stakeholders}

Using the prior distribution as a point of departure for substantive testing increases transparency towards the auditee because it requires auditors to be explicit about what information is incorporated into their analysis, and under which assumptions this information is translated into a probability distribution. We believe that this results in two concrete benefits for the auditee.

Firstly, the prior distribution encourages the auditor to quantify their assurance at the highest possible level of the organization as much as possible (de Swart, Wille, and Majoor 2013). Business processes at the top of the auditee's organization often provide assurance over processes lower in the organization. Therefore, by spending more effort on obtaining assurance from top-level processes the auditor can achieve a substantial increase in efficiency in a later stage of the audit, potentially lowering audit fees for the auditee. This is also beneficial for the auditee because it places the auditor in a position where they are able to suggest concrete improvements to the auditee at the highest levels of the organization, for 
example to improve efficiency in later audits. Moreover, the auditee may be inspired to conduct audit activities to incorporate information obtained by the auditor in the audit themselves next year.

Secondly, the prior distribution enables the auditor to optimize their mix of internal controls, analytical procedures, and substantive testing every year. Importantly, the auditee is able to review, verify, or scrutinize the information and controls used by the auditor to construct the prior distribution. For example, the auditee can point out relevant controls to the auditor that have not been included in the prior distribution, or they can provide additional assurance to the auditor by performing analytical procedures themselves. From the perspective of the auditee, the use of the prior distribution by the auditor provides insight into the services paid for and provided.

In the following section, we give several examples of how to efficiently incorporate existing information and make the underlying assumptions explicit.

\section{Pro 3: The Prior Distribution Allows for Improved Estimation of the Misstatement (and can Reduce Sample Size)}

If the auditor can justify incorporating appropriate prior information, then this will result in an increasingly precise estimate of the misstatement due to the extra knowledge that is used in the estimation procedure. Moreover, if this prior information implies a reduction in risk profile, incorporating this information into the prior distribution will result in a reduction of the sample size needed to get to the desired assurance about $\theta$.

To illustrate, if a prior distribution is applied that contains almost no information about values of $\theta$ (e.g., the $\operatorname{Beta}(1,1)$ prior distribution in Figure 1), almost all information required to arrive at a reasonable assurance about $\theta$ comes from the information in the sample. Alternatively, if a prior distribution is applied that contains appropriate, risk- 
reducing, information about $\theta$, less information is needed from the sample to arrive at a reasonable assurance about $\theta$. The top panel in Figure 2 illustrates this interchangeability between the incorporated prior information and the information from substantive testing.

As an example, the bottom left panel displays the non-informative prior distribution and the posterior distribution from the previous section. After seeing an error-free sample of 58 transactions, the 95 percent posterior upper bound in this case is 4.9 percent. The bottom right panel shows a scenario in which the auditor has access to appropriate audit information, through which they constructed an informative $\operatorname{Beta}(1,19)$ prior distribution. In the scenario in which the informative prior is constructed, the auditor only needs to inspect a sample of 40 transactions, which amounts to a reduction of 18 transactions as compared to the scenario in which the non-informative prior was constructed. Note that in the informative scenario, combining the informative $\operatorname{Beta}(1,19)$ prior with the $\operatorname{Binomial}(k=0 \mid n=40, \theta)$ likelihood of the sample, the posterior distribution is again a $\operatorname{Beta}(1,59)$ distribution whose 95 percent upper bound lies at 4.9 percent.

The latter scenario demonstrates the added value of the prior information since the auditor requires less information from substantive testing to arrive at the same amount of assurance about $\theta$. Incorporating appropriate information into the prior distribution can, therefore, increase efficiency and transparency in the audit by coherently reducing the required sample size in substantive testing. The assumptions behind a non-informative prior distribution are quick to justify, but the absence of information contained therein results in a larger sample size than may be necessary.

\section{Con 1: Justification of the Prior Distribution Takes Time and Effort}

An informative prior distribution is efficient in the sense that it allows for improved estimation of the misstatement and a potential reduction in sample size. However, the auditor 
must realize that the information that is incorporated into the prior distribution needs to be justified. Hence, the more information that is incorporated into the prior, the more work is required to substantiate why the incorporated information is appropriate for the population, and how it is incorporated into the prior distribution. Attention should be given to the amount of time and effort it takes to substantiate the information in the prior distribution for a substantial reduction in audit work versus the time and effort it takes to perform the reduction in audit work. Performing the Bayesian analysis makes most sense if the profit of the Bayesian analysis — the time and effort that is saved by reducing in sample size- outweighs the costs - the time and effort it takes to justify the Bayesian analysis.

Whether the pros of constructing a prior distribution outweigh its cons depends on the time and effort it takes to select and audit an extra sample. If the costs of selecting an extra sample exceed the time and effort to set up an informative prior distribution, a Bayesian analysis can be a profitable alternative to a frequentist analysis. For example, suppose that the auditor is performing an on-site indoor air quality audit (Asadi, Gameiro da Silva, and Costa 2013) for the auditee's office buildings around the world. They have the choice of either taking a large sample or performing an analytical procedure to construct the prior distribution. The analytical procedure in question correlates a building's air quality with its energy consumption, data which is available in digital format to the auditor. Of course, constructing the prior distribution on the basis of this correlation is not a trivial task and might take substantial time and effort. However, because the auditee has offices all around the world, traveling to — and inspecting — each building takes a significant amount of time and money. In these cases, it is likely that the possible reduction in sample size achieved by the information from the correlation analysis outweighs the time and effort that goes into specifying the prior distribution. Even if the auditor has easy access to the auditee and therefore decides that constructing the prior distribution is not worth the time and effort, they 
can fall back to a prior distribution that incorporates no existing information. The advantage of the Bayesian approach is that it provides the auditor with the flexibility to choose either of these options.

Finally, we are aware that the prior distribution is often perceived as difficult to construct and that this can be seen as a con as well. However, in the next section we attempt to resolve this by showing five methods of constructing a prior distribution. The specification of the prior distribution is complex because the available information must be represented by a probability distribution. Earlier work on prior distributions in an audit context has mainly focused on eliciting one directly from auditors' professional judgment (Abdolmohammadi 1985; Abdolmohammadi 1987). For instance, Chesley (1978) asked auditors to assign probabilities to specific values of $\theta$ (i.e., by eliciting a cumulative probability function). A reversed method was examined by Crosby (1981), who asked auditors to assign specific values of $\theta$ to probabilities (i.e., by eliciting values for the first, second, and third quartile of a probability density function). Such methods allow for coherent integration of expert knowledge about $\theta$ into the analysis, but require that the auditor has an advanced understanding of statistical concepts (e.g., probability distributions), something that is not necessarily part of an auditor's core expertise.

In the following section, we provide alternatives to such expert elicitation methods by discussing how various other sources of audit information can be formally incorporated into the prior distribution. The proposed methods provide a logical translation from audit information to a prior distribution, thereby making the specification of the prior distribution less daunting and complex for the auditor. Moreover, we have implemented these methods in statistical software so that they are available to auditors who are not experts on statistics but are willing to use the software approved by their technical office. This way, constructing a prior distribution on the basis of their already existing information requires no additional time 
and effort from the auditor. We show that these methods have the potential to increase audit efficiency and transparency.

\section{INTEGRATING INFORMATION INTO THE PRIOR DISTRIBUTION}

We discuss five methods for integrating existing information into the prior distribution. The first method is not informative about the characteristic $\theta$, whereas the other four methods integrate four types of information about $\theta$ into corresponding prior distributions: information regarding the prior probability of (in)tolerable misstatement occurring in the population, information from earlier (implicit) samples, historical information from last year's audit, and information from analytical procedures. For ease of explanation, the following methods assume that the auditor does not expect to find any misstatements in the sample. Note that the calculations are very similar when misstatements are expected (the resulting sample sizes would of course be different).

\section{Method 1: No Explicit Information}

The auditor can refrain from expressing an explicit opinion about $\theta$ by incorporating as little information as possible in the prior distribution, so that the resulting posterior distribution relies solely on the information from the sample (Blocher 1981; Martel-Escobar et al. 2018). Consider the scenario in which the auditor has no access to any existing information about which values of $\theta$ are more plausible a priori. Then, a suitable prior distribution is the previously introduced $\operatorname{Beta}(1,1)$ distribution as it assigns equal prior

probability mass to all values of $\theta$, and the posterior mode (most likely error) is $\frac{k}{n}$, which is equal to that of a frequentist approach (Albert 2003; Tuyl et al. 2008). 
Although the $\operatorname{Beta}(1,1)$ distribution is often a default choice due to its connection to frequentist methodology and its ease of application, it implies a conservative prior opinion about $\theta$ with respect to the upper bound of tolerable misstatement in the population. More specifically, the $\operatorname{Beta}(1,1)$ distribution expresses the prior opinion that intolerable misstatement is highly likely to occur in the population. To illustrate, for an upper bound of tolerable misstatement of five percent, the $\operatorname{Beta}(1,1)$ prior distribution assumes that, with 95 percent probability, the total misstatement in the population is larger than the upper bound of tolerable misstatement. If this reflects the auditor's true existing information about the misstatement in the population, a full inspection might arguably be a better choice.

By choosing a flat distribution no information is incorporated into the prior distribution. Therefore, the $\operatorname{Beta}(1,1)$ prior distribution is not advised when the auditor has access to information about the characteristic $\theta$. However, it can be useful in situations where the auditor, for conservative reasons, wants to specifically refrain from expressing an opinion about $\theta$, wants to retain some of the properties of a frequentist analysis, or when the auditor wants to have a benchmark analysis for a more informed Bayesian analysis.

\section{Example to Detect Overstatements}

Suppose the auditor must audit a new client and wants to determine the misstatement $\theta$ in a population. Because they are auditing a new client and do not have access to existing information about this population, they want to state a non-informed opinion with respect to $\theta$. Since the prior distribution contains no information about $\theta$, there is no need for the auditor to justify the information in the prior distribution.

Prior to calculating a sample size, the auditor makes an assessment of the expected (tolerable) errors in the sample. Assuming a $\operatorname{Beta}(1,1)$ prior distribution, and applying Bayes' theorem to find the minimum sample size $n_{\text {Method } 1}$ such that if no errors are detected 
in the sample, the posterior distribution has a 95 percent upper bound of five percent, the auditor finds $n_{\text {Method } 1}=58$. Using the $\operatorname{Beta}(1,1)$ prior is slightly more efficient than a frequentist analysis, in which the auditor must inspect $n_{\text {freq }}=60$ observations to reduce the sampling risk sufficiently (AICPA 2017). However, the posterior most likely error resulting from a $\operatorname{Beta}(1,1)$ prior distribution is equal to that of a frequentist analysis, and is 0 after seeing this error-free sample.

\section{Method 2: Information about the Probability of Misstatement}

The auditor can incorporate existing information regarding the prior probability of (in)tolerable misstatement occurring in the population in the prior distribution on $\theta$. In contrast to the expected misstatement, the probability of misstatement represents the auditor's assessment about how likely it is that the population contains material misstatement. Auditors generally state their opinion on the population misstatement using the posterior most likely error (ISA 450, ISA 530) after they have assessed that their work was sufficient for such statement by comparing the upper bound of the posterior distribution to the performance materiality $\theta_{\max }$ (ISA 320 ; IAASB 2018). We propose to use the value of $\theta_{\max }$ as an anchoring point for the prior distribution. Anchoring the prior distribution on the performance materiality allows the auditor to exploit the areas under the prior distribution above and below $\theta_{\max }$. These areas respectively express the prior information about the probability of the population misstatement being tolerable or not before a sample is selected and analyzed.

When using a beta distribution, the area under the prior distribution that lies below the performance materiality $P_{-}=\int_{0}^{\theta_{\max }} \frac{\theta^{\alpha-1}(1-\theta)^{\beta-1}}{B(\alpha, \beta)} d \theta$ quantifies the prior probability of tolerable misstatement occurring in the population, while the area that lies above the performance materiality $P_{+}=\int_{\theta_{\max }}^{1} \frac{\theta^{\alpha-1}(1-\theta)^{\beta-1}}{B(\alpha, \beta)} d \theta$ quantifies the prior probability of intolerable misstatement occurring in the population. Given the auditor's existing information 
about the probability of (in)tolerable misstatement occurring in the population, and given the expected misstatement in the sample, the $\alpha$ and $\beta$ parameters of the beta prior distribution are defined.

To illustrate how anchoring works in this context, suppose the auditor sets the performance materiality at five percent $\left(\theta_{\max }=0.05\right)$. Furthermore, they expect to find no misstatements in the sample (implying that the prior parameter $\alpha=1$ ) and assume a priori that tolerable misstatement is equally plausible to occur in the population as intolerable misstatement (implying that $P_{-}=P_{+}=0.5$ ). Since the prior probability of (in)tolerable misstatement is specified by the area under the prior distribution, equal prior probabilities can be achieved by setting the median of the beta distribution to the performance materiality $\theta_{\max }$. When $\alpha=1$, the median of the beta distribution can be expressed as $\theta_{\max }=\theta_{.50}=$ $1-2^{-\frac{1}{\beta}}$ (Kerman 2011). Consequently, the $\beta$ parameter of the prior distribution can be determined as $\beta=\frac{\ln \left(P_{+}\right)}{\ln \left(1-\theta_{\max }\right)}=\frac{\ln (0.5)}{\ln (1-0.05)}=13.51$. Therefore, the auditor's existing information regarding the prior probability of (in)tolerable misstatement occurring in the population is incorporated into a $\operatorname{Beta}(1,13.51)$ distribution.

\section{Example to Detect Overstatements}

We will now apply this method to a practical example from Harrison et al. (2002). The auditee has an internal control where software verifies the general ledger for transactions created by employees in the field. The auditor has information that on days that show a high sales activity across the country, one of the employees can disable the control system to get orders processed faster. To gain assuring evidence that the general ledger contains no material misstatement, the auditor might perform substantive testing of transactions that were recorded on days where the control was disabled. Suppose the auditor finds that this control is a critical 
check for 60 percent of all sales transactions, and so 60 percent of sales transactions made on the days that the system was disabled was not checked.

The prior distribution can be justified by the existing information that 60 percent of the transactions in the population might contain misstatement. Thus, the auditor wants to incorporate the existing information that the prior probability of intolerable misstatement is 60 percent. The auditor specifies $P_{+}=0.6$ and plans for zero tolerable deviations, thereby assuming a $\operatorname{Beta}(1,9.96)$ prior distribution.

Applying Bayes' theorem to find the sample size $n_{\text {Method } 2}$ such that if no errors are detected in the sample, the posterior distribution has a 95 percent upper bound of five percent, the auditor finds $n_{\text {Method } 2}=49$. Compared to the benchmark Bayesian analysis (method 1 ) this method results in a reduction in the sample size of $n=9$. Compared to the standard frequentist method where no prior information is incorporated the reduction in sample size amounts to $n=11$.

\section{Method 3: Information from Earlier Samples}

The auditor can incorporate information from a prescribed reduction in the required sample size in the prior distribution on $\theta$. Consider for example the scenario where the auditor can reduce their required sample size based on the risk assessment of the population, as determined by their audit guide. Since the information in the prior distribution can be interpreted as equivalent to that of an earlier sample, the auditor can use the reduction in sample size to construct their prior distribution (de Swart, Wille, and Majoor 2013; Steele 1992).

An application for this method can be found in the auditing standards, which state that the auditor may act on their collected audit information through the audit risk model (ARM) by reducing their required sample size in substantive testing (IAASB 2018). The ARM 
provides an association between the specified audit risk and the assessed risk of material misstatement, see Equation 2.

\section{Audit risk $=$ Inherent risk $\times$ Control risk $\times$ Detection risk}

According to the ARM, the audit risk is divided into three constituents: inherent risk, control risk, and detection risk. Inherent risk is the risk of a material misstatement due to an error in a financial statement before consideration of any related controls. Control risk is the risk of a material misstatement not prevented or detected by the internal control systems of the auditee (e.g., computer-managed databases). Detection risk is the risk that an auditor will fail to find material misstatements that exist in the auditee's financial statements. According to the ARM, the auditor is only involved in the detection risk and may adjust this accordingly to accommodate the other two risks so that the acceptable level of audit risk is retained. Therefore, a lower assessed risk of material misstatement may allow a higher tolerable detection risk and, in turn, requires less persuasive audit evidence (IAASB 2018). ISA 330 and ISA 530 prescribe that a lower quantity of evidence from substantive testing applies in these situations.

Currently, it is unclear how risk assessments can reduce the required sample size. Sampling manuals from audit firms and other institutions that perform substantive testing show a large variation in the extent to which reliance on risk assessments reduces the required sample size. Table 1 displays examples of the sample size reduction factor $R$ (Touw and Hoogduin 2012). To calculate $R$ we need -for a given upper bound of tolerable misstatement$n_{+}:$the largest sample size in the manual, and $n_{-}:$the smallest. The largest sample size $n_{+}$is often the same number, based on 95 percent confidence and zero expected misstatements in the sample. The smallest sample size $n_{-}$is based on the assumption of a low risk of material misstatement and a system of internal controls that functions well. Of course, the words 'low' and 'well' are subjective, and therefore, the number $n_{-}$varies when different audit sampling 
manuals are considered (see Table 1). By incorporating their risk assessments through the prior distribution, the auditor is able to make the relationship between these risk assessments and the required sample size explicit.

Table 1 shows that a reduction in sample size is common practice, and prescribed by the ISA. As explained at the beginning of this section, this reduction $\Delta n=n_{+}-n_{-}$can be incorporated in the prior distribution (assuming that the unseen samples contain no misstatements). More specifically, if the auditor expects no misstatements in the sample then $\alpha=1$. Next, $\Delta n$ can be incorporated into the beta prior distribution by setting the $\beta$ parameter of the prior distribution to $1+\Delta n$. For example, if the auditor, based on their assessments of inherent risk and control risk, has reduced the required sample by 20 transactions, this information is incorporated in a $\operatorname{Beta}(1,21)$ prior distribution.

Vice versa, instead of constructing the prior distribution based on a reduction in the sample size, the prior distribution can also be constructed on the basis of a sample that is already analyzed. Suppose that the auditor has taken the required sample containing 58 transactions, but found one deviation and therefore cannot conclude with 95 percent certainty that the maximum misstatement is lower than the performance materiality of five percent. The posterior distribution in this scenario is a $\operatorname{Beta}(2,59)$ distribution, which has its 95 th percentile at 7.6 percent. However, instead of judging that the population contains material misstatement, the auditor wants to perform additional testing on the population. By using the posterior distribution as a prior distribution for a second sample, the auditor can extend their analyzed sample without any penalty (Dienes 2011; Rouder 2014; Wagenmakers 2008). This is different than in a frequentist approach, where the auditor is unable to coherently extend testing of their analyzed sample while preserving the intended audit risk. Using the $\operatorname{Beta}(2,59)$ posterior distribution as a prior distribution, the auditor can maintain the required assurance on $\theta$ by inspecting 33 extra error-free samples from the population. These 33 
samples together with the 58 original samples coherently add up to $n=91$, the sample size that is required when using a Beta $(1,1)$ distribution and planning for one expected misstatement in the sample.

\section{Example to Detect Overstatements}

Suppose an auditor is testing for an upper bound of tolerable misstatement of five percent and is planning for zero expected misstatements in the sample. Furthermore, suppose that their audit guide prescribes a sample of 58 transactions when both inherent risk and control risk are high. However, the auditor has collected evidence that both inherent risk and control risk can be judged as medium instead of high. When both risks are judged as medium, their audit guide postulates that the required sample size for zero expected misstatements is 32.

The prior distribution can be justified by the reduction in required sample size. Using the reduction in the required sample size, the auditor specifies $\beta=1+\Delta n=27$, and arrives at a $\operatorname{Beta}(1,27)$ prior distribution.

Using the $\operatorname{Beta}(1,27)$ prior distribution, and applying Bayes' theorem to find the sample size $n_{\text {Method } 3}$ such that if no errors are detected in the sample, the posterior distribution has a 95 percent upper bound of five percent, the auditor again finds $n_{\text {Method } 3}=$ 32. Compared to the benchmark Bayesian analysis (method 1), the reduction in sample size is $n=27$. Compared to the standard frequentist method where no prior information is incorporated the reduction in sample size amounts to $n=29$.

\section{Method 4: Information from Last Year's Audit}

The auditor can incorporate information from last year's results in the prior distribution on $\theta$. A method for letting this historical information inform the current audit is suggested by van Batenburg and Kriens (1989), who consider last year's posterior distribution 
as a point of departure. If the result of last year's audit was positive, this posterior distribution generally has its 95 percent upper bound below the performance materiality. Assuming the auditor wants to exploit the information collected in year $t-1$ in the prior distribution for year $t$, it is reasonable to state a prior distribution on $\theta$ for year $t$ that is equal to the posterior distribution of year $t-1$.

Using the posterior distribution from year $t-1$ directly as a prior distribution for year $t$ implies that the estimated maximum misstatement in the population is already lower than performance materiality being the required maximum tolerable misstatement. The justification for such a prior distribution is grounded in the case where the population from year $t$ is the same as that of year $t-1$. No additional samples would be required to achieve the required assurance about $\theta$. Of course, the auditor shall have to decide the extent to which last year's population is compatible with that of this year. The assumption that the two are completely equivalent usually does not hold, as the population in the current year will only be comparable to that of the previous year to a certain extent. However, customer, product, quantity, and price of certain transactions might be equivalent, and the auditor can incorporate the extent of this equivalence in the prior distribution on $\theta$.

To quantify the auditor's knowledge on the equivalence of the populations in year $t$ and year $t-1$ a weighting factor $f$ can be introduced to the beta prior distribution (van Batenburg, O’Hagan, and Veenstra 1994). If the auditor finds that the two populations are comparable, they can assign a higher weight to the results of last year's audit and the value for $f$ should fall close to one. For example, if 70 percent of the transactions in the general ledger consists of transactions that are equivalent (with respect to the customer, product, quantity, and price) to those that were audited in last year's audit, the auditor can specify $f=0.7$. However, if they think that the populations are incomparable, the value for $f$ should fall close to zero. For example, suppose major errors were found in year $t-1$ after which the auditee 
implemented controls that make it very unlikely that these errors occur in year $t$. Since the major errors in the population of year $t-1$ are unlikely to occur in the population of year $t$, the information from year $t-1$ should be taken into account limitedly. This is reflected in a low prior probability for this information.

To illustrate this method, suppose the auditor has access to last year's posterior distribution $\operatorname{Beta}\left(\alpha_{t-1}, \beta_{t-1}\right)$, which has its 95 th percentile below the performance materiality. Given a value for $f$, the corresponding prior distribution can be determined as $\operatorname{Beta}\left(1+\left(\alpha_{t-1}-1\right) \times f, 1+\left(\beta_{t-1}-1\right) \times f\right)$.

\section{Example to Detect Overstatements}

Suppose last year the auditor performed substantive testing without incorporating any existing information. They inspected a full sample of 58 records and found that zero transactions were misstated. The posterior distribution from year $t-1$ is a $\operatorname{Beta}(1,59)$ distribution, which has its 95 th percentile at 0.049 . Consequently, the auditor inferred that, with 95 percent confidence, the misstatement in the population was below the upper bound of tolerable misstatement of five percent.

The prior distribution can be justified by the equivalence between the populations of last year and those of the current year. This year, the auditor has collected information that the populations of last year and this year are comparable to the extent of 60 percent $(f=0.6)$ since 60 percent of customers, products, quantity, and price are equivalent. New customers in the population (40 percent) have not been subject to the validated controls of last year. Therefore, the auditor specifies $f=0.6$ and determines $\beta=35.8$.

Using a Beta $(1,35.8)$ prior distribution, and applying Bayes' theorem to find the sample size $n_{\text {Method } 4}$ such that if no misstatements are detected in the sample, the posterior distribution has a 95 percent upper bound of 5 percent, the auditor finds $n_{\text {Method } 4}=23$. 
Compared to the benchmark Bayesian analysis (method 1) this method results in a reduction in the sample size of $n=35$. Compared to the standard frequentist method where no prior information is incorporated the reduction in sample size amounts to $n=37$.

\section{Method 5: Information from Analytical Procedures}

The auditor can incorporate information from analytical procedures (e.g., a benchmark analysis) in the prior distribution on $\theta$. In the previously discussed methods of constructing a prior distribution based on existing knowledge, we have described the procedure by which this knowledge can be translated into the prior distribution. However, there is no such clear procedure for information acquired from analytical procedures, since these procedures can vary strongly depending on the type of information that is incorporated in the prior. The following approach thus necessitates a stronger substantiation of its data and assumptions, and how these assumptions are incorporated into the prior distribution. However, given the increasing availability of big data from other sources than the general ledger, such as sensor data or financial data from external vendors, we foresee that the possibility to apply a wide range of analytical procedures will grow rapidly in the near future. Bayesian methods offer a way to stack these procedures and determine how much additional comfort is needed from substantive testing to come to a reasonable conclusion about $\theta$.

\section{Example to Detect Overstatements}

For example, Stringer and Stewart (1986) introduced statistical techniques to assess potential misstatements based on, for instance, regression models. A concrete example of such a model is benchmarking the relationship between sales and costs of sales within the auditee's industry sector. This relationship can be modeled by a linear equation:

$$
C=\beta_{0}+\beta_{1} \cdot S+\epsilon
$$


where $C$ and $S$ denote the auditee's Cost of Sales and Sales, respectively. In practice, this relationship is often more complex than is presented in Equation 3, and the auditor must carefully construct and evaluate the applied regression model. However, for ease of understanding we will continue our example with this simplified model.

After estimating the parameters $\beta_{0}$ and $\beta_{1}$ from a data set consisting of values of $C$ and $S$ for peer companies, the auditor must check the assumptions underlying their linear regression. If these assumptions hold, the prediction for the costs of sales of the auditee, given the actual sales of the auditee, can be derived in the form of a normal probability distribution. In a typical analytical procedure, this prediction of the cost of sales is summarized in the form of a 90 percent two-sided interval. Next, the auditor verifies whether the booked cost of sales by the auditee falls within the predicted interval. If it does, then less evidence from substantive testing is required than if the booked cost of sales falls outside of the predicted interval.

The procedure described above is a frequentist one. However, similar procedures within the Bayesian philosophy have been proposed before. For instance, Deakin and Granof (1974) and Kinney and Bailey (1976) noted that regression analysis can be used to revise the auditor's prior probabilities of the population being misstated or not by testing whether the auditee's booked cost of sales is different from the predicted cost of sales.

The prior distribution can be justified by the data and the auditee's numerical prediction of the cost of sales. In this analytical procedure, our proposal for the prior distribution on $\theta$ is to use the relative error distribution from the linear regression. The relative error distribution is the normal distribution $(\operatorname{Normal}(\mu, \sigma))$ that captures the uncertainty of the prediction of the cost of sales by means of the linear regression, scaled to be a percentage of the total cost of sales. The mean $\mu$ of the prior distribution on $\theta$ is the relative deviation of the auditee's booked cost of sales when compared to the predicted cost of sales 
according to the benchmark data $\frac{C-\hat{C}}{C}$. The standard deviation of the prior distribution on $\theta$, induced by the benchmark data, is expressed by the standard deviation of the distribution of $\epsilon$. We propose to use this distribution as the prior distribution on $\theta$ to quantify the amount of substantive testing needed on top of this analytical procedure to conclude that the audit risk is sufficiently small enough.

Suppose that the auditor is assessing the risk of including fraudulent bribery payments at the auditee's organization. To perform an audit of the payments, the auditor needs to investigate both the auditee's costs of sales and their actual sales. The sum of the booked costs of sales is $\$ 223,994,405$ and the sum of the sales is $\$ 298,112,312$, respectively. The allocated performance materiality has been set to $\$ 112,500$, or five percent of the booked cost of sales. The existing information is a benchmark of audited figures on the cost of sales and actual sales of peer companies $(n=100)$ in the auditee's industry group. These benchmark data are plotted in Figure 3.

The auditor estimates the parameters in Equation 3 using linear regression leading to $\beta_{0}=241,300$ and $\beta_{1}=0.7366$. This gives the following estimate $\hat{C}$ of the auditee's costs of sales:

$$
\hat{C}=\$ 241,300+0.7366 S=\$ 219,817,866 .
$$

The auditor confirms that $\epsilon$ is normally distributed having a standard deviation of $\sigma_{\epsilon}=$ $\$ 11,090,408$. Assuming that the benchmark data is representative for the auditee, the auditor can incorporate the information from this benchmark in the $\operatorname{Normal}(\mu, \sigma)$ prior distribution on $\theta$. The mean $\mu$ of the prior distribution on $\theta$ is the relative deviation of the auditee's booked costs of sales when compared to the predicted cost of sales according to the benchmark data $\frac{C-\hat{C}}{C}=\frac{223,994,405-219,817,866}{223,994,405}$, and equals 1.9 percent. The standard deviation 
$\sigma$ of the prior distribution on $\theta$ can be determined as $\frac{\sigma_{\epsilon}}{C}=\frac{11,090,408}{223,994,405}$, and is 5 percent. Since the auditor is focusing on overstatements only, the prior distribution is truncated to the interval $[0 ; 1]$. Finally, the auditor has arrived at the truncated $\operatorname{Normal}(0.019 ; 0.05)$ distribution as the prior distribution on $\theta$.

Applying Bayes' theorem to find the sample size $n_{\text {Method } 5}$ such that, if no errors are detected in the sample, the posterior distribution has a 95 percent upper bound of five percent, the auditor finds $n_{\text {Method } 5}=50$. Compared to the benchmark Bayesian analysis (method 1$)$ this method results in a reduction in the sample size of $n=8$. Compared to the standard frequentist method where no prior information is incorporated the reduction in sample size amounts to $n=10$.

Keeping in mind that there are in principle infinite possibilities to use all sorts of data and statistical learning methods to create prior distributions, this approach is much more generic than those in the previous sections. The only restriction on the statistical learning method used in the analytical procedure is that this method not only delivers a most likely value for the audited figure but also a probability distribution of the method's error. The commonly used linear regression certainly meets this requirement. However, by using validation samples next to training samples, even non-parametric methods can be equipped with a way to derive a probability distribution for the error, so we do not see this restriction as a severe one.

\section{Comparison of Posterior Distributions}

To illustrate the effect of incorporating the existing information from the previous subsections on the auditor's final judgment about $\theta$, Figure 4 shows each of the prior distributions (left panel) and their corresponding posterior distributions (right panel) after inspecting a sample of 30 transactions, of which zero transactions were misstated. The figure 
illustrates that prior distributions that incorporate increasingly stronger audit information (i.e., assign more prior probability mass to lower values of $\theta$ ) result in posterior distributions that assign more probability mass to lower values of $\theta$.

Table 2 shows the posterior mode, the corresponding 95 percent upper bounds of the posterior distributions, and the margin of error of the estimate (i.e., the difference between the upper bound and the mode of the posterior distribution). Compared to the benchmark Bayesian analysis in method 1, the other prior distributions result in a more precise estimate of the population misstatement. Moreover, as can be seen from the 95 percent upper bounds, only the posterior distribution described in method four has its 95th percentile below five percent, and thus supports the statement that the population misstatement is, with 95 percent probability, lower than the performance materiality of five percent. The prior distribution from this example incorporates relatively strong audit information since it assumes that 60 percent of all transactions in the population were equivalent to those of last year with respect to customer, product, quantity, and price. Table 2 also shows that the most likely error is often estimated to be zero, except for the most likely error given resulting from the prior constructed in method five. That is because, even though the prior distribution incorporated assuring audit information, it also assumed a prior most likely error of 1.9 percent.

\section{CONCLUDING COMMENTS}

Over the years, there has been a need for efficient use of expert knowledge and existing information in audit data analytics (Appelbaum et al. 2017; Chesley 1975). We have shown that applying Bayesian statistics allows for incorporation of this knowledge and information into the statistical analysis. Next, we have outlined five methodologies that auditors can use to incorporate this information into a prior distribution. We are convinced the 
auditor can only face the growing challenges of today's auditing field, ensuring maximum audit quality while maintaining high efficiency, by tailoring their audit specifically to the situation of the auditee. Bayesian statistics allows for coherent incorporation of many sources of acquired audit information into the statistical sampling procedure, allowing auditors to build on existing information, and offering them the flexibility to control how they arrange their activities to aggregate audit evidence over the audit as a whole. Especially in today's critically examined audits, Bayesian statistics provides a transparent and efficient manner of auditing.

For convenience, we have demonstrated the five methods for constructing a prior distribution using a beta distribution. However, the methods we have discussed are generic. When applied to a different situation (e.g., a gamma distribution), the specifics of the equations are different, but the incorporated prior information remains the same. A comparison of the beta and gamma distributions for MUS is beyond the scope of this article, and we refer to the work of Stewart (2013, pp. 62-70) for further reading. However, we have included illustrations of the discussed methods using a gamma distribution in the appendix to this article.

When drawing inferences in the Bayesian framework, the auditor has to consider that substantive test work, including sample sizes, can be reduced via a prior distribution. If a prior is weakly informative, the potential reduction in sample size is small. On the other hand, if a prior is very informative, the potential reduction in sample sizes is large. However, this large potential reduction in sample size comes at a cost, as the auditor needs to show, firstly, that the existing information is valid and relevant for the audit, and secondly that the translation of this information into the prior distribution is appropriate. We have discussed the tools to translate this existing information into the prior distribution. 
However, in practice the existing information is often prone to measurement error. To the extent that this measurement error exists, it is important that the auditor takes the quality of the information into account when constructing a prior distribution. It can be useful for the auditor to work together with a statistician to determine if, and how, the existing information should be translated into a probability distribution. As more complex information is incorporated into the analysis, the role of the statistician becomes increasingly important to assure that the information is incorporated accurately into the prior distribution.

In our approach, the auditor constructs the prior distribution on the basis of audit evidence (Methods $2-5$ ), which means that there is information to justify the prior distribution. In the case where this is not possible, the auditor is able to fall back to the trivial prior distribution which reflects no existing information (Method 1). Because in both these scenarios it is transparent how the prior distribution is constructed and what information it incorporates, it can be scrutinized by other stakeholders, (internal and external) reviewers, or team members. For example, transparency towards a regulator can be given in the scenario where the regulator is trying to trace the steps of the auditor, or wants to scrutinize the auditor's judgment.

However, to mitigate critique that the auditor can reason to a foregone conclusion based on the specific choice of prior distribution they can assess the robustness of their outcomes to the choice of the prior distribution. This can be done via a sensitivity analysis (Hoijtink et al. 2019; Liu and Aitkin 2008), where the auditor considers the amount of achieved assurance or estimated maximum misstatement for different prior specifications. Sensitivity analyses have been proposed before in auditing (Martel-Escobar, Vázquez-Polo, and Hernández-Bastida 2005), as well as in other scientific fields, to see of evidence for a particular scenario is relatively stable across a range of prior beliefs, suggesting that the statistical analysis yields conclusive results in multiple scenarios. The auditor can assess this 
sensitivity to the prior distribution by changing its parameters, or by changing the family of the prior distribution. For example, the auditor can compare their informative $\operatorname{Beta}(\alpha, \beta)$ prior distribution with the benchmark $\operatorname{Beta}(1,1)$ prior distribution, or calculate the results using a gamma prior distribution.

We are aware that, despite its broad use in the audit practice, strong arguments against the use of the Audit Risk Model (ARM) as shown in method 3 have been formulated. The main objection is that the constituents of the audit risk influence each other, and should therefore not be treated as independent probabilities (Cushing and Loebbecke 1983; Jiambalvo and Waller 1984; Kinney 1983; Leslie 1984). Nonetheless, the ARM is widely used and accepted in practice. We would like to highlight the fact that we do not attempt to criticize the validity of the ARM. In fact, in Method 3 we build on the ARM by transforming assessments of inherent risk and control risk into a prior distribution. We view it as a successful model demonstrating a methodological approach to structuring audit risk (Arzhenovskiy et al. 2019).

Finally, we have attempted to eliminate the difficulty of auditors determining the proper distributional family, specifying the prior, conducting sensitivity analyses, and documenting these choices, by implementing these methods in the R package "jfa" (Derks 2021) and JASP for Audit, a module for the freely available and open-source statistical software program JASP that is designed to facilitate Bayesian statistical auditing (Derks, de Swart, Wagenmakers, Wille, and Wetzels 2019). We hope that, by implementing these methods in a digital environment for which no extensive programming knowledge is required, they can be of aid to researchers and auditors interested in using Bayesian methods in audit sampling. 


\section{REFERENCES}

Abdolmohammadi, M. J. 1985. Bayesian inference research in auditing: Some methodological suggestions. Contemporary Accounting Research 2 (1): 76-94.

Abdolmohammadi, M. J. 1987. Bayesian inference in auditing: Evidence on the most appropriate assessment techniques. Accounting and Business Research 17 (68): 291300.

Abeysekera, I. 2015. Student preferences for instructional methods in an accounting curriculum. International Journal of Teaching and Learning in Higher Education 27 (3): $310-319$.

Albert, M. 2003. Bayesian rationality and decision making: A critical review. Analyse \& Kritik 25 (1): 101-107.

Asadi, E., M. C. G. Da Silva, and J. J. Costa. 2013. A systematic indoor air quality audit approach for public buildings. Environmental Monitoring and Assessment 185: 865875.

American Institute of Certified Public Accountants (AICPA). 2017. Appendix C: Monetary Unit Sampling Tables. In Audit Guide: Audit Sampling (pp. 141-145). John Wiley \& Sons, Ltd.

Appelbaum, D., A. Kogan, and M. A. Vasarhelyi. 2017. Big data and analytics in the modern audit engagement: Research needs. Auditing: A Journal of Practice \& Theory 36 (4): $1-27$.

Arzhenovskiy, S. V., A. V. Bakhteev, T. G. Sinyavskaya, and N. N. Hahonova. 2019. Audit risk assessment model. International Journal of Economics and Business Administration 7 (1): 74-85. 
Batenburg, P. C. V., and J. Kriens. 1989. Bayesian discovery sampling: A simple model of Bayesian inference in auditing. Journal of the Royal Statistical Society. Series D (The Statistician) 38 (4): 227-233.

Batenburg, P. C. V., A. O’Hagan, and R. H. Veenstra. 1994. Bayesian discovery sampling in financial auditing: A hierarchical prior model for substantive test sample sizes. Journal of the Royal Statistical Society. Series D (The Statistician) 43 (1): 99-110.

Beaulieu, P. R. 2001. The effects of judgments of new clients' integrity upon risk judgments, audit evidence, and fees. Auditing: A Journal of Practice \& Theory 20 (2): 85-99.

Beck, P. J., I. Solomon, and L. A. Tomassini. 1985. Subjective prior probability distributions and audit risk. Journal of Accounting Research 23 (1): 37-56.

Blocher, E. 1981. Assessment of prior distributions: The effect on required sample size in Bayesian audit sampling. Accounting and Business Research 12 (45): 11-20.

Brivot, M., M. Roussy, and M. Mayer. 2018. Conventions of audit quality: The perspective of public and private company audit partners. Auditing: A Journal of Practice \& Theory 37 (2): 51-71.

Broeze, E. 2006. Validation of Risk Assessments in Auditing. Ph.D. dissertation. VU University Amsterdam. Amsterdam: Limpberg Instituut.

Brown-Liburd, H., H. Issa, and D. Lombardi. 2015. Behavioral implications of big data's impact on audit judgment and decision making and future research directions. Accounting Horizons 29 (2): 451-468.

Carpenter, B, A. Gelman, M. D. Hoffman, D. Lee, B. Goodrich, M. Betancourt, M. Brubaker, J. Guo, P. Li, and A. Riddell. 2017. Stan: A probabilistic programming language. Journal of Statistical Software 76 (1): 1-32. 
Chesley, G. R. 1975. Elicitation of subjective probabilities: A review. The Accounting Review 50 (2): $325-337$.

Chesley, G. R. 1978. Subjective probability elicitation techniques: A performance comparison. Journal of Accounting Research 16 (2): 225-241.

Chan, H., and W. Smieliauskas. 1990. Further tests of the modified moment bound in audit sampling of accounting populations. Auditing: A Journal of Practice \& Theory 9 (3): 167-182.

Christensen, B. E., R. J. Elder, and S. M. Glover. 2015. Behind the Numbers: Insights into Large Audit Firm Sampling Policies. Accounting Horizons 29 (1): 61-81.

Coram, P., J. Ng, and D. Woodli. 2003. A survey of time budget pressure and reduced audit quality among Australian auditors. Australian Accounting Review 13 (29): 38-44.

Corless, J. C. 1972. Assessing prior distributions for applying Bayesian statistics in auditing. The Accounting Review 47 (3): 556-566.

Crosby, M. A. 1980. Implications of prior probability elicitation on auditor sample size decisions. Journal of Accounting Research 18 (2): 585-593.

Crosby, M. A. 1981. Bayesian statistics in auditing: a comparison of probability elicitation techniques. The Accounting Review 56 (2): 355-365.

Cushing B. E., and J. K. Loebbecke. 1983. Analytical approaches to audit risk: A survey and analysis. Auditing: A Journal of Practice \& Theory 3 (1): 23-41.

Deakin, E. B., and M. H. Granof. 1974. Regression analysis as a means of determining audit sample size. The Accounting Review 49 (4): 764-771.

Derks, K. (2021).jfa: Bayesian and Classical Audit Sampling. R package version 0.5.3. 
Derks, K., J. de Swart, E.-J. Wagenmakers, J. Wille, and R. Wetzels. 2019. JASP for audit: Bayesian tools for the auditing practice. doi: 10.31234/osf.io/9f6ub

de Swart, J., J. Wille, and B. Majoor. 2013. Het 'push left'-principe als motor van data analytics in de accountantscontrole [the 'push-left'-principle as a driver of data analytics in financial audit]. Maandblad voor Accountancy en Bedrijfseconomie 87: 425-432.

Dienes, Z. 2011. Bayesian versus orthodox statistics: Which side are you on? Perspectives on Psychological Science 6 (3): 274-290.

Earley, C. E. (2015). Data analytics in auditing: Opportunities and challenges. Business Horizons 58 (5): 493-500.

Elder, R. J., A. D. Akresh, S. M. Glover, J. L. Higgs, and J. Liljegren. 2013. Audit sampling research: A synthesis and implications for future research. Auditing: A Journal of Practice \& Theory 32 (1): 99-129.

Etz, A. 2018. Introduction to the concept of likelihood and its applications. Advances in Methods and Practices in Psychological Science 1 (1): 60-69.

Felix, W. L. 1976. Evidence on alternative means of assessing prior probability distributions for audit decision making. The Accounting Review 51 (4): 800-807.

Gepp, A., M. K. Linnenluecke, T. J. O’Neill, and T. Smith. 2018. Big data techniques in auditing research and practice: Current trends and future opportunities. Journal of Accounting Literature 40: 102-115.

Godfrey, J., and J. Neter. 1984. Bayesian bounds for monetary unit sampling in accounting and auditing. Journal of Accounting Research 22 (2): 497-525. 
Harrison, K. E., R. P. Srivastava, and R. D. Plumlee. 2002. Auditors' evaluations of uncertain audit evidence: Belief functions versus probabilities. In Belief functions in business decisions (pp. 161-183). Physica. Heidelberg.

Hitzig, N. B. 1995. Audit sampling: A survey of current practice. The CPA Journal 65 (7): 54-57.

Hoekstra, R., R. D. Morey, J. N. Rouder, and E.-J. Wagenmakers. 2014. Robust misinterpretation of confidence intervals. Psychonomic Bulletin \& Review 21 (5): $1157-1164$.

Hoijtink, H., J. Mulder, C. van Lissa, and X. Gu. 2019. A tutorial on testing hypotheses using the Bayes factor. Psychological methods 24 (5): 539-556.

International Auditing and Assurance Standards Board (IAASB). 2018. Handbook of International Quality Control, Auditing Review, Other Assurance, and Related Services Pronouncements, Volume I. New York: International Federation of Accountants.

Jiambalvo J., and W. Waller. 1984. Decomposition and assessments of audit risk. Auditing: A Journal of Practice \& Theory 3 (2): 80-88.

Kachelmeier, S. J., T. Majors, and M. G. Williamson. 2014. Does intent modify risk-based auditing? The Accounting Review 89 (6): 2181-2201.

Kerman, J. (2011). A closed-form approximation for the median of the beta distribution. arXiv preprint arXiv:1111.0433.

Kinney, W. 1975. Decision theory aspects of internal control system design/compliance and substantive tests. Journal of Accounting Research (Supplement) 13: 14-29.

Kinney W. 1983. A note on compounding probabilities in auditing. Auditing: A Journal of Practice \& Theory 2 (2): 13-22. 
Kinney, W., and A. D. Bailey. 1976. Regression analysis as a means of determining audit sample size: A comment. The Accounting Review 51 (2): 396-401.

Knoblett, J. A. 1970. The applicability of Bayesian statistics in auditing. Decision Sciences 1 (3-4): 423-440.

Kruschke, J. K., and T. M. Liddell. 2017. Bayesian data analysis for newcomers. Psychonomic Bulletin \& Review 25 (1): 155-177.

Laws, D. J., and A. O’Hagan. 2002. A hierarchical Bayes model for multilocation auditing. Journal of the Royal Statistical Society: Series D (The Statistician) 51 (4): 431-450.

Lee, L., W. Kerler, and D. Ivancevich. 2018. Beyond excel: Software tools and the accounting curriculum. AIS Educator Journal 13 (1): 44-61.

Leslie D. A. 1984. An analysis of the audit framework focusing on inherent risk and the role of statistical sampling in compliance testing. In Proceedings of the 1984 Touche Ross University of Kansas Symposium on Auditing Problems, eds. H. Stettler, and N. Ford, 126-132. Lawrence, KA: School of Business, University of Kansas.

Lindley, D. V. 2004. That wretched prior. Significance 1 (2): 85-87.

Liu, C. C., and M. Aitkin. 2008. Bayes factors: Prior sensitivity and model generalizability. Journal of Mathematical Psychology 52 (6): 362-375.

Lombardi, D., R. Bloch, and M. Vasarhelyi. 2014. The future of audit. Journal of Information Systems and Technology Management 11 (1): 21-32.

Maingot, M., and T. K. Quon. 2009. Sampling practices of internal auditors at corporations on the standard \& poor's Toronto stock exchange composite index. Accounting Perspectives 8 (3): 215-234.

Martel-Escobar, M., F.-J. Vazquez-Polo, and A. Hernandez-Bastida. 2005. Analysing the 
independence hypothesis in models for rare errors: an application to auditing. Journal of the Royal Statistical Society: Series C (Applied Statistics) 54 (4): 795-804.

Martel-Escobar, M., F.-J. Vazquez-Polo, and A. Hernandez-Bastida. 2018. Bayesian inference in auditing with partial prior information using maximum entropy priors. Entropy 20 (12): 919-928.

Meeden, G. 2003. A Bayesian solution for a statistical auditing problem. Journal of the American Statistical Association 98 (463): 735-740.

Neter, J., J. K. Loebbecke. 1975. Behaviour of Major Statistical Estimators in Sampling Accounting Populations-An Empirical Study. New York: American Institute of Certified Public Accountants.

Neter, J., and J. K. Loebbecke. 1977. On the behavior of statistical estimators when sampling accounting populations. Journal of the American Statistical Association 72 (359): 501-507.

Neter, J., and K. James. 1975. Auditing research monograph No. 2. Behavior of major statistical estimators in sampling accounting populations. AICPA. New York.

Power, M. K. 1992. From common sense to expertise: Reflections on the prehistory of audit sampling. Accounting, Organizations and Society 17 (1): 37-62.

Ramage, J. G., A. M. Krieger, and L. L. Spero. 1979. An empirical study of error characteristics in audit populations. Journal of Accounting Research 17: 72-102.

Rouder, J. N. 2014. Optional stopping: No problem for Bayesians. Psychonomic bulletin \& review 21 (2): 301-308.

Srivenkataramana, T. 2018. Application of statistical sampling to audit and control. DHARANA-Bhavan's International Journal of Business 12 (1): 14-19. 
Steele, A. 1992. Audit risk and audit evidence: The Bayesian approach to statistical auditing. London: Academic Press.

Stewart, T. 2013. A Bayesian audit assurance model with application to the component materiality problem in group audits. Ph.D. dissertation. VU University Amsterdam. Amsterdam: Limpberg Instituut.

Stewart, T., L. Strijbosch, H. Moors, P. van Batenburg. 2007. A simple approximation to the convolution of gamma distributions. CentER Discussion Paper, No. 2006-27, ISSN 0924-7815, Revised Version Sept. 2007.

Stringer, K. W., and T. R. Stewart. 1986. Statistical techniques for analytical review in auditing. John Wiley \& Sons.

Sahu, S. K., and T. M. F. Smith. 2006. A Bayesian method of sample size determination with practical applications. Journal of the Royal Statistical Society: Series A (Statistics in Society) 169 (2): 235-253.

Swinamer, K., M. Lesperance, and H. Will. 2004. Optimal bounds used in dollar-unit sampling: A comparison of reliability and efficiency. Communications in StatisticsSimulation and Computation 33 (1): 109-143.

Titera, W. R. 2013. Updating audit standards enabling audit data analysis. Journal of Information Systems 27 (1): 325-331.

Touw, P., and L. Hoogduin. 2012. Statistiek voor audit en controlling. Academic Service. Tsui, K.-W., E. M. Matsumura, and K.-L. Tsui. 1985. Multinomial-Dirichlet bounds for dollar-unit sampling in auditing. Accounting Review 60: 76-96.

Tuyl, F., R. Gerlach, and K. Mengersen. 2008. A comparison of Bayes-laplace, Jeffreys, and other priors: The case of zero events. The American Statistician 62 (1): 40-44. 
van der Nest, D., L. Smidt, and D. Lubbe. 2015. The application of statistical and/or nonstatistical sampling techniques by internal audit functions in the South African banking industry. Risk Governance and Control: Financial Markets \& Institutions 5 (1): $71-80$.

Wagenmakers, E.-J., M. Lee, T. Lodewyckx, and G. J. Iverson. 2008. Bayesian versus frequentist inference. In Bayesian evaluation of informative hypotheses (pp. 181-207). Springe, New York, NY.

Yoon, K., and T. Pearce. 2020. Can substantive analytical procedures with data and data analytics replace sampling as tests of details? Journal of Emerging Technologies in Accounting. 


\section{APPENDIX}

The $\operatorname{Gamma}(\alpha, \beta)$ distribution has a shape parameter $\alpha$ and a rate (inverse scale) parameter $\beta$ that determine its shape. The gamma prior distribution is a common choice for monetary unit sampling, in which a sample of $n$ transactions containing a total of $k$ proportional errors (taints) is selected from a population of $M$ monetary units. The $\operatorname{Gamma}(\alpha, \beta)$ distribution is combined with the $\operatorname{Poisson}\left(\lambda=k \frac{M}{n} \mid \theta\right)$ likelihood to form a $\operatorname{Gamma}\left(\alpha+k, \beta+\frac{1}{M / n}\right)$ posterior distribution. The most likely error (mode) of the distribution, given $\alpha \geq 1$, is $\frac{\alpha-1}{\beta}$.

\section{Method 1: No Explicit Information}

The posterior distribution resulting from a $\operatorname{Gamma}(1,0)$ prior distribution depends solely on the information in the sample since its posterior most likely error is $k \frac{M}{n}$. This specific prior distribution is improper, however, meaning that gives an infinite amount of probability mass to positive values of $\theta$.

\section{Method 2: Information about the Probability of Misstatement}

For the gamma distribution, the areas corresponding to tolerable and intolerable misstatement, respectively, are:

$$
P_{-}=\int_{0}^{\theta_{\max }} \frac{\beta^{\alpha}}{\Gamma(\alpha)} \theta^{\alpha-1} e^{-\beta \theta} d \theta \quad P_{+}=\int_{\theta_{\max }}^{M} \frac{\beta^{\alpha}}{\Gamma(\alpha)} \theta^{\alpha-1} e^{-\beta \theta} d \theta
$$

Given an assessment of the probability of tolerable misstatement $P_{+}=1-P_{-}$and planning for zero expected deviations ( $\alpha=1)$, the $\beta$ parameter of the gamma prior distribution can be determined as $\beta=-\frac{\ln \left(P_{+}\right)}{\theta_{\max }}$. The prior distribution is therefore a $\operatorname{Gamma}\left(1,-\frac{\ln \left(P_{+}\right)}{\theta_{\max }}\right)$ distribution. 


\section{Method 3: Information from Earlier Samples}

Considering the reduction in required sample size $\Delta n=n_{+}-n_{-}$, the $\beta$ parameter of the gamma distribution can be set to $\beta=\frac{1}{M / \Delta n}$. The prior distribution is therefore a $\operatorname{Gamma}\left(1, \frac{1}{M / \Delta n}\right)$ distribution.

\section{Method 4: Information from Last Year's Audit}

Suppose the auditor has access to last year's posterior $\operatorname{Gamma}\left(\alpha_{t-1}, \beta_{t-1}\right)$ distribution, which has its 95 percent upper bound below the performance materiality. Given a value for $f$, the prior distribution is a $\operatorname{Gamma}\left(1+\left(\alpha_{t-1}-1\right) \times f, \beta_{t-1} \times f\right)$ distribution. 


\begin{tabular}{lc}
\hline & $R$ \\
\hline Big 4 office & 15 \\
NL Public body & 2.7 \\
EU Public body & 3 \\
NL Tax and Customs & 8 \\
NL Tier2 office & 3 \\
\hline
\end{tabular}

Table 1 . The sample size reduction factor $R=\frac{n_{+}}{n_{-}}$in various audit manuals. 


\begin{tabular}{|c|c|c|c|c|c|}
\hline Method & Prior & Posterior & Posterior Mode & Posterior 95\% Upper Bound & Margin of Error \\
\hline 1 & $\operatorname{Beta}(1,1)$ & $\operatorname{Beta}(1,31)$ & 0 & 0.0921 & 0.0921 \\
\hline 2 & $\operatorname{Beta}(1,9.96)$ & $\operatorname{Beta}(1,39.96)$ & 0 & 0.0722 & 0.0722 \\
\hline 2 & $\operatorname{Beta}(1,27)$ & $\operatorname{Beta}(1,57)$ & 0 & 0.0512 & 0.0512 \\
\hline 4 & $\operatorname{Beta}(1,35.8)$ & $\operatorname{Beta}(1,65.8)$ & 0 & 0.0445 & 0.0445 \\
\hline 5 & Truncated Normal $(0.019,0.05)$ & NA & 0.0243 & 0.0660 & 0.0417 \\
\hline
\end{tabular}

${ }^{1}$ The margin of error is the difference between the posterior mode and the posterior $95 \%$ upper bound (upper bound - mode).

Table 2. Description of prior distributions and the corresponding posterior distributions after taking a sample of $n=30, k=0$. Each of the $\operatorname{Beta}(\alpha, \beta)$ prior distributions is combined with a $\operatorname{Binomial}(k=0 \mid n=30, \theta)$ likelihood to form a $\operatorname{Beta}(\alpha+k, \beta+n-k)$ posterior distribution. The truncated $\operatorname{Normal}(\mu, \sigma)$ prior distribution has no analytical solution for the combination with a $\operatorname{Binomial}(k=0 \mid n=30, \theta)$ likelihood and the posterior distribution is therefore determined via HMC sampling. The mean of the HMC samples is given as the posterior mode. The margin of error is the difference between the posterior mode and the posterior 95\% upper bound. 
- - Prior distribution — Posterior distribution

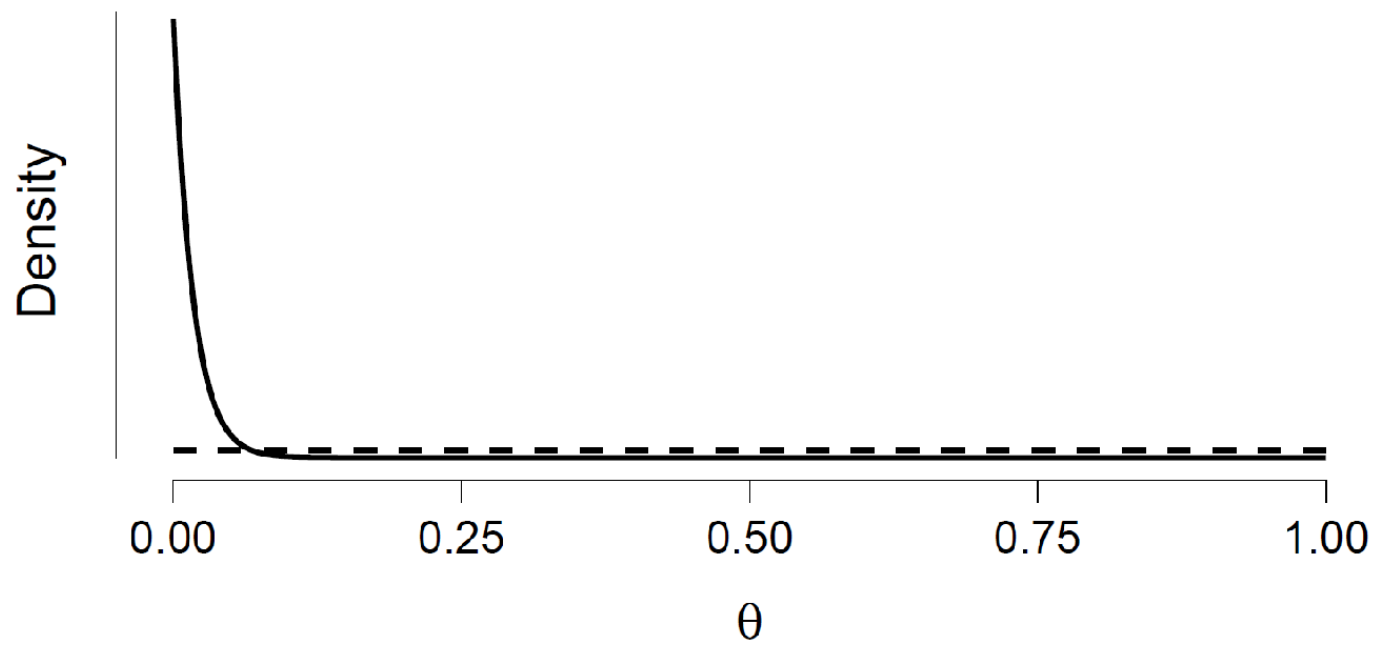

Figure 1: Example of a $\operatorname{Beta}(1,1)$ prior distribution on the population misstatement proportion $\theta$. The information in the prior distribution is weighed with the information in the sample, the $\operatorname{Binomial}(k=0 \mid n=58, \theta)$ likelihood, to form the $\operatorname{Beta}(1,59)$ posterior distribution. 


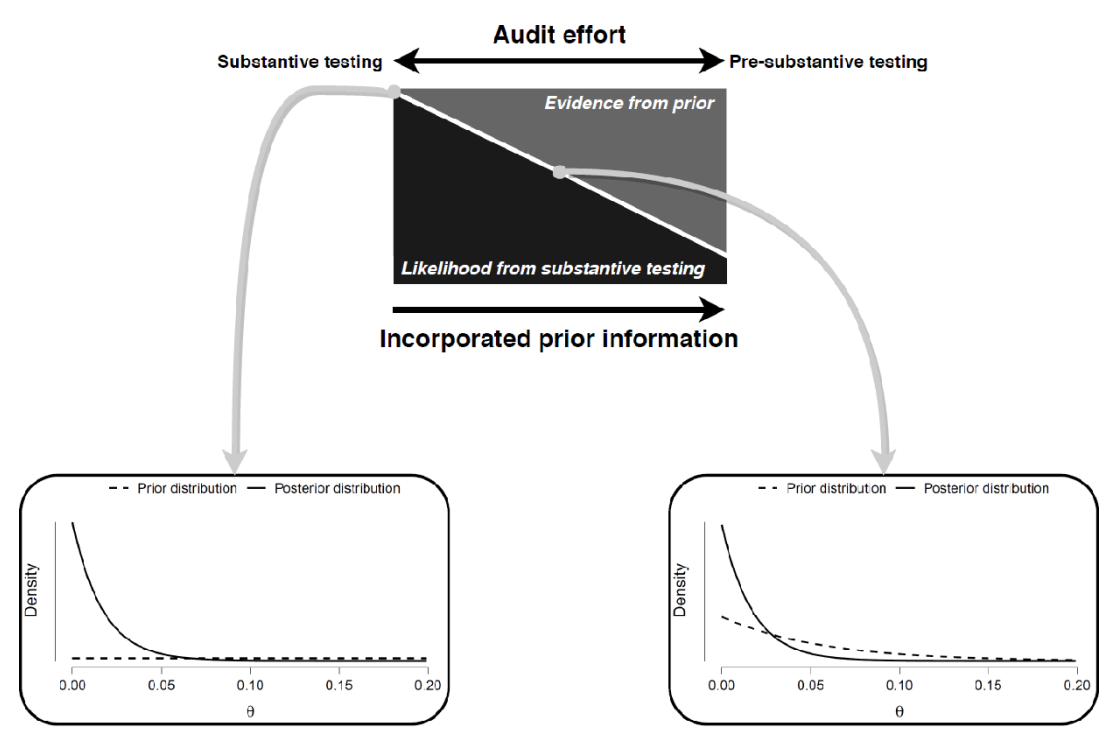

Figure 2: The top panel illustrates the balance between the amount of incorporated prior information and the required information (likelihood) from substantive testing. The $\operatorname{Beta}(1,1)$ prior distribution in the bottom left panel contains little prior information and combined with a $\operatorname{Binomial}(k=0 \mid n=58, \theta)$ likelihood, forms a $\operatorname{Beta}(1,59)$ posterior distribution. The Beta $(1,19)$ prior distribution in the right panel contains more information and therefore requires less information from a sample to reach the same assurance. Combined with a $\operatorname{Binomial}(k=0 \mid n=40, \theta)$ likelihood, the posterior distribution is again a $\operatorname{Beta}(1,59)$ distribution. 


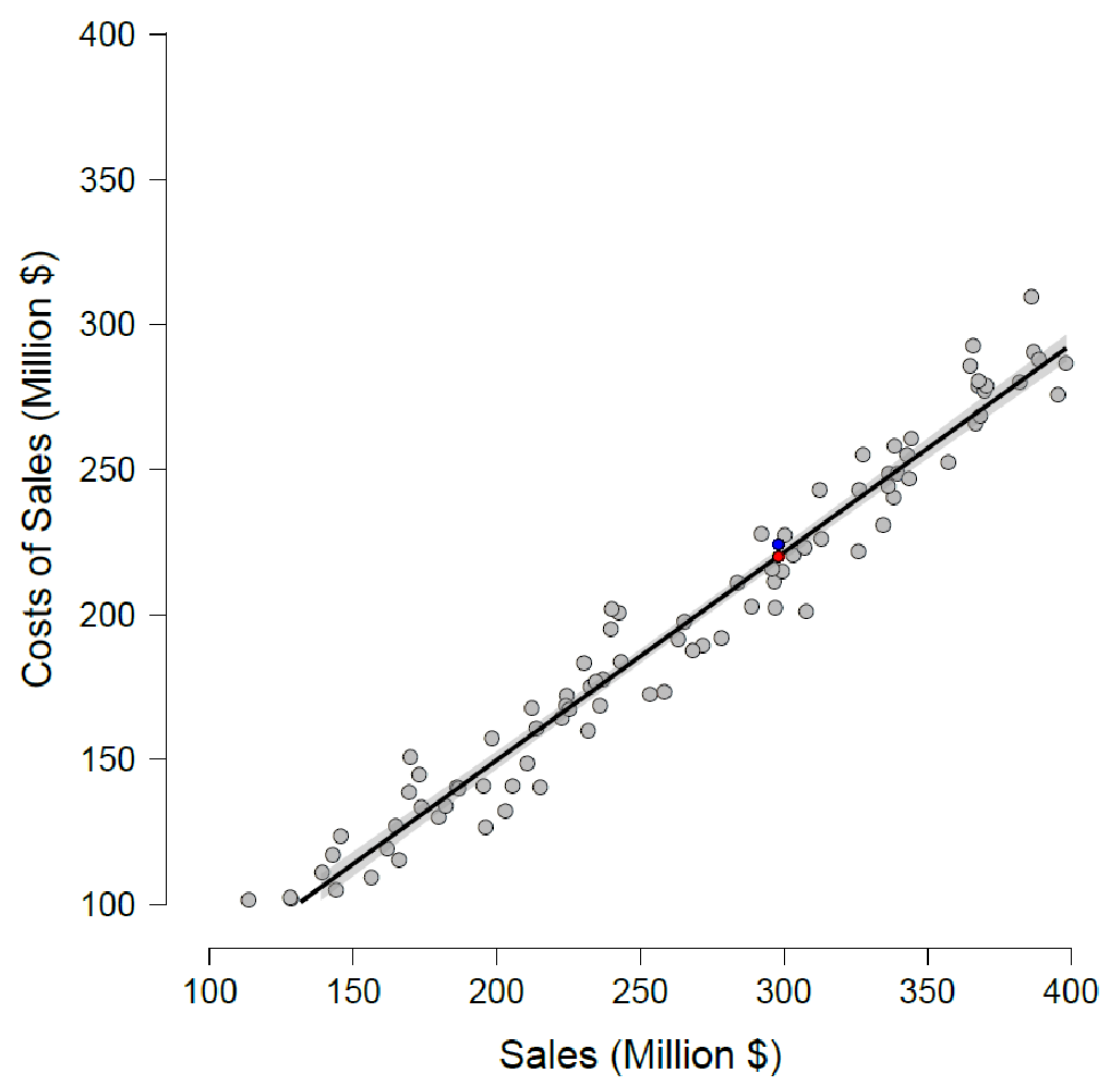

Figure 3: Scatter plot of the cost of sales $C$ (million \$) versus the actual sales $S$ (million \$) for peer companies of the auditee. The blue and red dot indicate the auditee's booked and expected costs of sales based on the benchmark, respectively. 


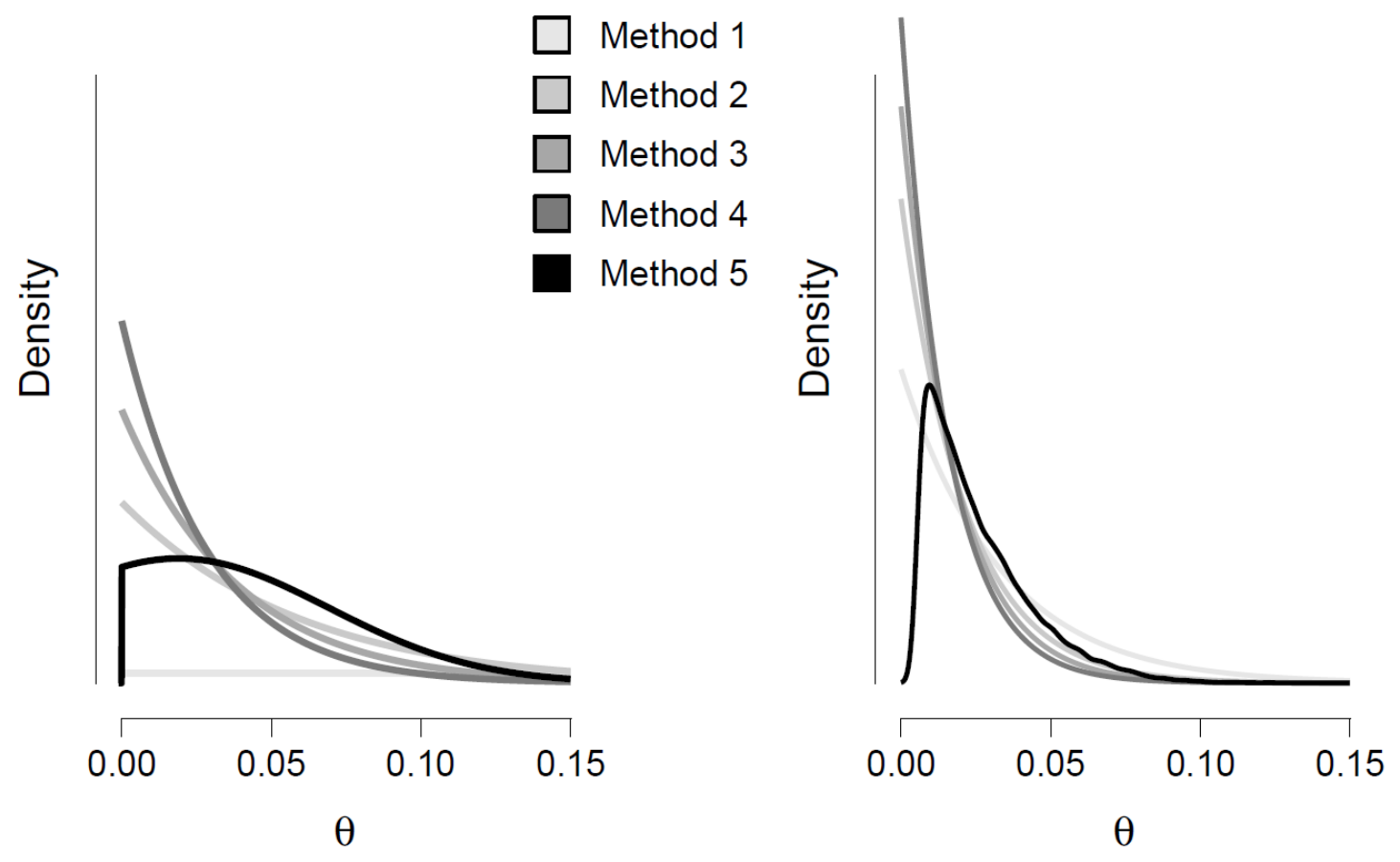

Figure 4: Prior distributions (left panel) and posterior distributions (right panel) for each of the five discussed methods after inspecting a sample of $n=30$ transactions, of which $k=0$ contained a misstatement. As can be seen from the figure, prior distributions that initially assign more probability mass to lower values of $\theta$ also result in posterior distributions that assign more probability mass to lower values of $\theta$. 